\title{
Inflammatory Rhetoric Aside, Is There Anything to Learn From Fed Is Best?
}

\section{Editorial}

I recently read an upsetting blog post on Forbes.com: Fed Is Best Foundation Says WHO Breastfeeding Guidelines Fail To Meet Human Rights Standards. According to this article, babies were starving because of the Baby-Friendly Hospital Initiative (BFHI). By "imposing" BFHI on families, the breastfeeding community was violating families' human rights. They further stated that there was no evidence to support BFHI. In fact, BFHI was a public health emergency. Policymakers need to do something right now!

Fed Is Best has no research to support their claims, but they have stories of babies who had problems. These are important, but an anecdote is not evidence. (In contrast, a quick search on PubMed yielded almost 800 studies on the BFHI.) However inflammatory their rhetoric, I cannot dismiss Fed Is Best completely out of hand. Fed Is Best has identified some areas where our service to mothers could improve. Let's examine some of those issues and consider the history of the BFHI.

\section{History of BFHI}

As many of you know, the BFHI was launched worldwide following the 1991 Innocenti Declaration. The goals were originally aspirational. UNICEF's Executive Director, James Grant (UNICEF, 2003) asked practitioners with expertize in breastfeeding and child survival what factors would increase breastfeeding rates worldwide.

The aims of BFHI are to promote baby- and motherfriendly maternity facilities to encourage, respect, and support each mother's informed choice of mode of feeding and caring for her infant(s), and to ensure exclusive breastfeeding in the hospital and beyond. (Cox, 2010)

The goal was to increase infant survival, and the original 10-steps evolved from this discussion and the Innocenti conference. Since that time, the 10-steps have been updated as the science evolves. The 10-steps are not set in stone, but are a living document. I have no doubt that they will continue to change, while still being true to the original goals.

\section{Challenges}

Those sound like good goals. If that is the case, what is fueling the more strident anti-breastfeeding rhetoric? Here are a few of the challenges I've identified.
Share this:

\section{Rooming In}

I recently read a post that claimed that rooming-in was unkind to mothers because it interferes with their sleep. This is why some hospitals still think nurseries are a good idea. So what are the issues here? Babies who room-in have higher rates of exclusive breastfeeding (Labbok, Taylor, \& Parry, 2013). Further, exclusively breastfeeding mothers get more sleep (Kendall-Tackett, Cong, \& Hale, 2011). Why the contradiction? I think it's matter of simple mechanics. Babies in the U.S. room-in in bassinets, rather than the lovely sidecars they have in some hospitals in the UK. As Tully and Ball's research has demonstrated, mothers breastfeed more when their babies are in sidecars rather than in bassinets next to the bed (Tully \& Ball, 2012). The physical action required to get a baby from bassinet to mother's hospital bed can be very difficult, especially for mothers who had cesareans or assisted vaginal deliveries. They either need to get out of bed, or need to twist and pull to bring the baby to them. Ideally, they would have some help. But they may be alone in their room, and they'd need to completely wake up to complete this operation.

Honestly, the rooming-in mothers probably are more tired. Some mothers report that rooming-in seems cruel when they are exhausted. There are some safety concerns as well. Exhausted mothers may drop their babies as they try to grab them in the middle of the night.

We need to listen to these concerns. The answer is not to eliminate rooming-in, but to make it easier for mothers to get their babies. Sidecars on hospital beds, or something similar, would be a supportive practice.

\section{Early Feeding and Failure to Thrive}

Fed Is Best was founded because a mother had low production that no one identified, and her infant was harmed as a result. The way to address this gap is twofold. First, we must identify mothers at risk for low milk production. These include physiological and mechanical issues, such as insufficient glandular tissue, previous surgery or breast injury, or polycystic ovary syndrome.

Another potential factor, which is far more common and thus concerning, is birth interventions. Depression and breastfeeding difficulties are fellow travelers: where 
you see one, you are likely to see the other. And both have been specifically linked to interventions such as synthetic oxytocin, epidurals, and pain medications (Kendall-Tackett, Cong, \& Hale, 2015; Uvnas-Moberg, 2015). All of these interventions specifically block naturally occurring oxytocin, which impacts ability to breastfeed. In some cases, these interventions are necessary and/or are what families want. Parents are often told that these interventions have no negative effect. That is simply not true. As with any medical intervention, there are risks and benefits that need to be weighed. One of the risks is the potential for more breastfeeding problems.

If we see mothers postpartum, they may have already had these interventions. We need to know that these interventions can impact lactogenesis II and milk production by blocking oxytocin. But we also need to strike a delicate balance. We don't want to communicate to mothers that we think they will fail, which we can do if we imply that they may have difficulties. We can watch them without communicating that we think they will fail.

We also need to empower mothers by teaching them to know when they have enough milk. Keeping a simple diaper $\log$ is the easiest way. Mothers can make themselves crazy with trying to clock all the details, such as time at the breast, letdowns, foremilk/hindmilk, and so forth. Monitoring output (i.e., baby poop) is still the best way to keep track. Poop color won't change if babies aren't getting enough to eat. And if mothers have any concerns, they should be able to touch base with someone who can check.

\section{Lack of Support}

The 10th step of the BFHI is that mothers will have support in the community. This is where I think we are the weakest. Mothers leave the hospitals and face a pastiche of breastfeeding support services. This system can be very difficult to navigate and mothers often founder with lack of support. The sharp drop-off in exclusive breastfeeding rates suggests that this continues to be a significant problem.

This is, in no way, a shot at the professionals and volunteers working to provide support. I meet them all across the U.S. and I know they are working as hard as they possibly can, often with very limited resources. When budget cuts come, lactation is often the first program that goes. I consider this more of a systemic problem. If we are serious about increasing exclusive breastfeeding past the hospital stay, and doing it in a way that is safe for mothers and babies, our institutions and health organizations need to commit the resources to make sure it's possible. That means money! There are some communities where this is happening, so I know it's possible. Let's make it happen for all mothers.

\section{Listening to Mothers}

The most successful implementation of BFHI policies have occurred when there is community buy-in. Mothers feel like they are part of the process and that these goals have not been imposed from the outside. When I collected mothers' stories for the special issue on tongue-tie, the striking similarity was that these mothers struggled in pain, with faltering milk production-often for weeks-and no one listened. I could see why they were frustrated.

Mothers' concerns are important. Perhaps, as we move to the next phase, we can incorporate mothers' feedback about what they perceive as the challenges. Let's also make sure our hospital practices are mother-friendly as well as baby-friendly. That may limit the use of birth interventions that impede breastfeeding and make the hospital a safer place for mothers to be. Some studies have incorporated mothers' perspectives. But there is enough information floating around on social media to suggest that we might be missing some things that mothers find important. Let's think about how we can incorporate this feedback.

In my opinion, Fed Is Best has not accurately characterized the work of the breastfeeding community. But this organization would not have taken off if they weren't tapping into some discontent. If we can, let's set aside our ire and use this as an opportunity to improve practice.

Thanks for all your extraordinary efforts on behalf of our mothers and babies. You are making a difference every day.

Kathleen Kendall-Tackett, PhD, IBCLC, RLC, FAPA

Editor-in-Chief

\section{References}

Cox, S. (2010). Altering hospital maternity culture: Current evidence for the ten steps to successful breastfeeding. Amarillo, TX: Praeclarus Press. 
Kendall-Tackett, K., Cong, Z., \& Hale, T. W. (2011). The effect of feeding method on sleep duration, maternal well-being, and postpartum depression. Clinical Lactation, 2(2), 22-26. http:// dx.doi.org/10.1891/215805311807011593

Kendall-Tackett, K., Cong, Z., \& Hale, T. W. (2015). Birth Interventions related to lower rates of exclusive breastfeeding and increased risk of postpartum depression in a large sample. Clinical Lactation, 6(3), 87-97. http://dx.doi.org/10.1891/2158-0782.6.3.87

Labbok, M., Taylor, E., \& Parry, K. (2013). Achieving exclusive breastfeeding: Translating research into action. Amarillo, TX: Praeclarus Press.
Tully, K. P., \& Ball, H. L. (2012). Postnatal unit bassinet types when rooming-in after cesarean birth: Implications for breastfeeding and infant safety. Journal of Human Lactation: Official Journal of International Lactation Consultant Association, 28(4), 494-505. http://dx.doi.org/10.1177/ $\underline{0890334412452932}$

UNICEF. (2003). James P. Grant biography. Retrieved from https://www.unicef.org/about/who/index bio grant $\underline{\mathrm{html}}$

Uvnas-Moberg, K. (2015). Oxytocin: The biological guide to motherhood. Amarillo, TX: Praeclarus Press.

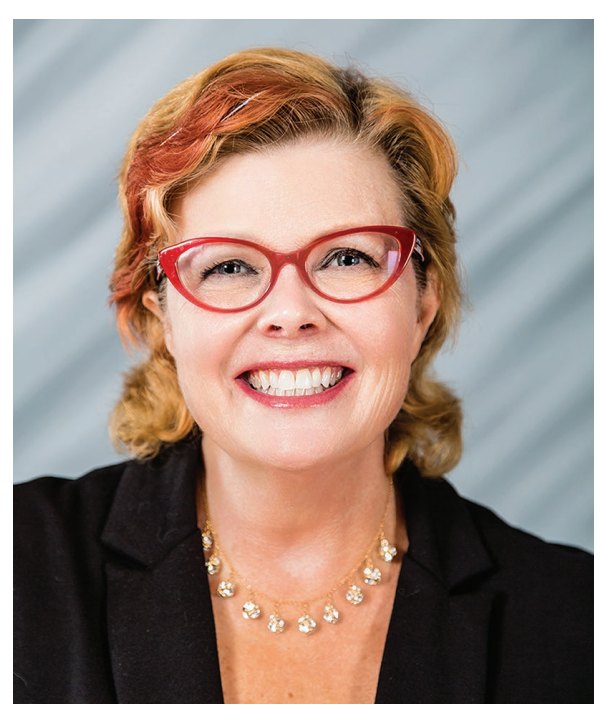

Dr. Kendall-Tackett, PhD, IBCLC, RLC, FAPA, is a health psychologist, an IBCLC, and the owner and editor-in-chief of Praeclarus Press, a small press specializing in women's health. Dr. Kendall-Tackett is editor-in-chief of two peerreviewed journals: Clinical Lactation and Psychological Trauma. Dr. Kendall-Tackett specializes in women's health research including breastfeeding, depression, trauma, and health psychology, and has won many awards for her work including the 2017 President's Award for Outstanding Service to the Field of Trauma Psychology from the APA's Division 56.

\section{Cultural Sensitivity for Health Care Professionals App Launch, From TJC}

The Joint Commission has released a mobile application featuring information from the Cultural Sensitivity: A Pocket Guide for Health Care Professionals reference guide:

\section{https://www.jcrinc.com/cultural-sensitivity-a-pocket-guide-for-health-care-professionals-second-edition/}

Available in a printed version designed to fit neatly into a lab coat pocket or as a mobile app, the reference guide offers healthcare professionals the information they need to successfully communicate with patients while respecting their specific cultural needs and expectations related to health care. The second edition focuses on the pregnancy and birth, pediatric, and end-of-life needs related to cultural customs. The app covers the following cultures: African American, Anglo American, Asian, Hispanic/Latino, Middle Eastern, Native American, Russian, South Asian, and Southeast Asian.

Source: USBC 